Background The aim of this study was to develop and externally validate a prognostic model for survival in people living with HIV/AIDS (PLWHA) initiating ART based on two large population-based cohorts in China.

Approach The derivation cohort consisted of PLWHA treated between February 2004 and December 2019 in a tertiary center in Guangzhou, South China, and validation cohort of patients treated between February 2004 to December 2018 in another tertiary hospital in Shenyang, Northeast China. We assessed 20 candidate predictors for an endpoint of death from all causes. The prognostic model was developed from a multivariable cox regression model with predictors selected using the least absolute shrinkage and selection operator (Lasso). To assess the model's predictive ability, we quantified the discriminative power using the concordance (C) statistic and calibration accuracy by comparing predicted survival probabilities with observed survival probabilities estimated with the Kaplan-Meier method.

Outcomes The derivation cohort included 16481 patients and the external validation cohort comprised 5751 participants. The final model included 10 predictors: age, body mass index, route of HIV acquisition, coinfection with tuberculosis, coinfection with hepatitis C virus, haemoglobin, CD4 cell count, platelet count, aspartate transaminase, and plasma glucose. The C-statistic was 0.84 (95\% confidence interval 0.82-0.85) in internal validation after adjustment of optimism and 0.84 $(0.82-0.87)$ in external validation, which remained consistently above 0.75 in all landmark time points within five years of follow up when using time-updated laboratory measurements. The calibration accuracy was satisfactory in both derivation and validation cohorts.

Innovation and Significance We developed and externally validated a model to predict long-term survival in PLWHA on ART, which could be applied to individualized patient counseling and management during treatment, and future innovative trial design.

\section{P349 DEMOGRAPHIC AND BEHAVIOURAL CHARACTERISTICS OF PATIENTS DIAGNOSED WITH EARLY VERSUS LATE SYPHILIS AT WESTERN SYDNEY SEXUAL HEALTH CENTRE, 2015-2019}

${ }^{1} Y$ Hughes*, ${ }^{1} \mathrm{C}$ Chung, 1,2,3 Zablotska-Manos, 1,2,3 D Lewis. 'Western Sydney Sexual Health Centre, Parramatta, Australia; ${ }^{2}$ Westmead Clinical School, Westmead, Australia; ${ }^{3}$ Marie Bashir Institute for Infectious Diseases and Biosecurity, Westmead, Australia

10.1136/sextrans-2021-sti.396

Background Western Sydney Sexual Health Centre (WSSHC) provides a free sexual health service for its culturally-diverse population. As local syphilis incidence is increasing, we determined demographic and behavioural characteristics associated with early syphilis infection at WSSHC.

Methods We conducted a 5-year retrospective study of WSSHC's syphilis cases (2015-2019). Patient and laboratory data were extracted from medical records for analysis. The study definition of early syphilis (ES) included cases of primary syphilis, secondary syphilis, early latent infection $(<2$ years) and latent syphilis of unknown duration (VDRL titre $\geq 1: 4$ ). Late syphilis (LS) included both cases acquired $>2$ years previously and latent syphilis cases of unknown duration (VDRL titre $\leq 1: 2$ ). Statistical methods employed the Chisquare test for trend (diagnoses over time) and univariate/ multivariate logistic regression analyses to identify ES-associated variables. The study received local Ethics Committee approval.

Results 557 cases of syphilis, including 380 (68\%) ES and 177 (32\%) LS cases, were diagnosed in 553 individuals (454 men, 99 women). Syphilis diagnoses increased over time; early syphilis cases increased $26 \%$ annually $(\mathrm{p}<0.001)$. Median age (years) was $32(\mathrm{IQR}=19-17)$ for $\mathrm{ES}$ patients and 39 $(\mathrm{IQR}=20-86)$ for LS patients. ES patients, when compared with LS patients, were more likely to be male $(338 / 380$ [89\%] vs. $120 / 177$ [68\%];OR 3.82, 95\%CI=2.44-6.00), <35 years old (226/286 [79\%] vs. 154/271 [57\%];OR 2.86, 95\% $\mathrm{CI}=1.97-4.15)$, Australia-born (213/380 [56\%] vs. 43/177 [24\%];OR 3.97, 95\%CI $=2.67-5.92)$, previously infected with syphilis $(66 / 380 \quad[17 \%]$ vs. $10 / 177 \quad$ [6\%];OR $3.51, \quad 95 \%$ $\mathrm{CI}=1.76-7.01)$, chemsex-users $(72 / 380$ [19\%] vs. 4/177 [2\%]; OR 10.11, 95\%CI=3.63-28.17), PrEP-users (70/327 [21\%] vs. $6 / 170 \quad[4 \%] ; \mathrm{OR} \quad 7.44,95 \% \mathrm{CI}=3.16-17.54)$ and $\mathrm{HIV}$ coinfected $\quad(53 / 380 \quad[14 \%] \quad$ vs. $7 / 177 \quad$ [4\%];OR $4.83, \quad 95 \%$ $\mathrm{CI}=2.14-10.89$ ). Multivariable regression modelling indicated that these same ES-associated variables remained significant in men $(\mathrm{p}<0.05)$.

Conclusions Male gender, age $<35$ years, Australia-born, use of chemsex or PrEP, HIV co-infection and previous syphilis infection are risk factors associated with ES at WSSHC.

\section{P350 INFLUENCE OF THE COVID-19 PANDEMIC ON THE EPIDEMIOLOGY AND RESISTANCE OF NEISSERIA GONORRHOEAE IN AUSTRIA}

U Fuernkranz*, M Haller, A Stary. Pilzambulatorium Schloesselgasse, Vienna, Austria

\subsection{6/sextrans-2021-sti.397}

Background Gonorrhoea is a health threat, infection levels and drug resistances increased over the last decades. The COVID19 pandemic that hit Austria in 2020 led to several lockdown events such as closing of bars, clubs, hotels and the order to stay at home. The current study was designed to elucidate the influence of these measures in order to curtail the pandemic on the number of $\mathrm{N}$. gonorrhoeae diagnosed and their respective resistance patterns.

Methods Retrospective data of patients positive for N. gonorrhoeae and resistances to ceftriaxone, cefixime, azithromycin and penicillin were compared using data from 2019 and 2020 collected in and sent to the Outpatients Centre for Infectious Venero-dermatological Diseases in Vienna. Resistance testing was carried out by means of the Etest ${ }^{\circledR}$; MIC values were interpreted according to EUCAST guidelines.

Results Numbers of patients tested positive for N. gonorrhoeae declined dramatically from 639 in 2019 to 381 in 2020. The most prominent reduction was during the first lockdown (March to May 2020), with half as many gonococcal infections compared to this period in 2019. The second lockdown (November/December 2020) did not result in significantly lower numbers of gonococcal infected patients. In both years, no ceftriaxone-resistant isolate was detected. However, $2.3 \%$ of isolates from women showed borderline susceptibility (MIC $=0.094$ and $0.125 \mu \mathrm{g} / \mathrm{ml}$ ), compared to $0.4 \%$ of isolates from men. During the two years of evaluation, cefixime and azithromycin resistant strains decreased (4\% to $0 \%$ and $19 \%$ to $7 \%$, respectively), while penicillin-resistant strains increased from $9 \%$ to $20 \%$. 
Conclusion The first lockdown resulted in a decrease of $62.7 \%$ of infected individuals. A remarkable decrease of resistant gonococcal strains to azithromycin and no resistance to cefixime occurred in 2020. This is in contrast to an increase of resistances until 2019. Increasing numbers of isolates of women approached the MIC breakpoint for ceftriaxone.

\section{P351 CLINICAL PRESENTATIONS OF SYPHILIS DIAGNOSED AT WESTERN SYDNEY SEXUAL HEALTH CENTRE, 2015-2019}

${ }^{1} Y$ Hughes*, ${ }^{1} \mathrm{C}$ Chung, ${ }^{1,2,3} \mid$ Zablotska-Manos, ${ }^{1,2,3} \mathrm{D}$ Lewis. 'Western Sydney Sexual Health Centre, Parramatta, Australia; ${ }^{2}$ Westmead Clinical School, Westmead, Australia; ${ }^{3}$ Marie Bashir Institute for Infectious Diseases and Biosecurity, Westmead, Australia

\subsection{6/sextrans-2021-sti.398}

Background Correct syphilis staging requires attentive historytaking and clinical examination; this assists with optimal patient management. We describe the clinical presentations of syphilis cases diagnosed at Western Sydney Sexual Health Centre (WSSHC) over a five-year period.

Methods We undertook a retrospective descriptive study of syphilis presentations at WSSHC between January 2015 and December 2019. Paper and electronic medical records were reviewed for 557 syphilis cases managed during this period. Clinical data were extracted and entered into an Access database for subsequent analysis. The Chi square test for trend was used to analyse incident infections over time. Ethics Committee approval was obtained.

Results During the study period, there were 203 symptomatic (93 primary, 110 secondary) and 354 asymptomatic (351 latent, 3 late neurosyphilis) syphilis cases. Primary and secondary syphilis cases increased over time $(\mathrm{p}=0.038$ and $\mathrm{p}=0.004$, respectively). Most primary $(91 / 93,98 \%)$ and secondary $(99 / 110,90 \%)$ cases were in men. Ulceration was a feature of 92/93 (99\%) primary syphilis cases; one additional patient presented with isolated inguinal lymphadenopathy. Locations of syphilitic ulceration were as follows: penile (68, $74 \%)$, anal $(19,21 \%)$, male genital skin $(4,4 \%)$ and oral $(4$, $4 \%)$. Most ulcers were solitary $(65 / 93,70 \%)$ although $30 \%$ (27/92) were multiple. Most of the secondary syphilis cases presented with a rash $(84 / 110,76 \%)$; the rash was demonstrable on palms/soles in 34/84 (40\%) cases and on the trunk in $73 / 84(87 \%)$ cases. Palmoplantar rash alone occurred in $7 / 84$ $(8 \%)$. Oral lesions and condylomata lata were documented in $16(15 \%)$ and 10 (9\%) of secondary syphilis patients, respectively. Six (5\%) secondary syphilis patients also had CSF-confirmed neurosyphilis; three were symptomatic (headache, $n=2$; neck stiffness, $\mathrm{n}=1$; blurred vision/photophobia, $\mathrm{n}=1$; reduced hearing, $\mathrm{n}=1$ ).

Conclusions Syphilis presents in many different ways and correct staging requires careful examination in order to inform clinical management and partner notification practice.

\section{P354 LABORATORY CAPACITY ASSESSMENT FOR SEROLOGICAL DETECTION OF SYPHILIS IN GUANGDONG, CHINA}

1,2L Yiwen*, ${ }^{2} \mathrm{Q}$ Xiaolin, ${ }^{1,2} \mathrm{Z}$ Heping. ${ }^{1}$ Dermatology Hospital of Southern Medical University, Guangzhou, China; ${ }^{2}$ Southern Medical University, Guangzhou, China

10.1136/sextrans-2021-sti.399
Objective To investigate and identify the detection capability of syphilis in the STD (sexually transmitted disease) laboratories in Guangdong, China.

Method An External quality assessment (EQA) of syphilis tests was performed and an online questionnaire surveys on tests and screening algorithms were conducted. Three positive and two negative EQA panels were prepared for qualitative or quantitative test of Nontreponemal tests (NTT) and Treponemal test (TT). The samples were distributed to all participants in October 2019 and the results were reported within one month and analyzed by Guangdong Central STD Laboratory through an online quality assessment software.

Results A total of 838 laboratories participated in the program, which come from different STD clinics, including primary, secondary and tertiary general hospitals, Maternal and Child Health Care Hospitals and CDC from the provincial, prefecture and county levels in Guangdong. The results showed that overall coincidence rate of the 838 participated laboratories was $98.0 \%$. The coincidence rate of NTT were that $99.3 \%$ of qualitative test and $96.7 \%$ of quantitative test. Coincidence rate of qualitative TT were 99.3\%. There were 286 laboratories reported the quantitative TT results, with an overall coincidence rate of $94.7 \%$. Toluidine red unheated serum test (TRUST) was the most used NTT, and Treponema pallidum particle assay (TPPA) popularly chosen as Treponemal test. There were 273 laboratories returned a second survey on syphilis screening algorithms. Among them, 118(43.2\%) laboratories adopted the traditional screening, 81(29.7\%) laboratories adopted the reverse screening, and 64(23.4\%) laboratories employed two algorithms simultaneously. Moreover, 10(3.7\%) laboratories adopted the ECDC (European Centre for Disease Control and Prevention) algorithm.

Conclusion Laboratories in Guangdong Province have great capabilities of syphilis diagnosis, especially in the CDC and the Maternal and Child Hospitals. Traditional screening is the most used algorithm, while the use of reverse screening is increasing.

\section{P355 THE PERFORMANCE OF LABORATORY-BASED DIAGNOSTIC ASSAYS TO DIAGNOSE PRIMARY SYPHILIS CASES AT WESTERN SYDNEY SEXUAL HEALTH CENTRE, 2015-2019}

${ }^{1} Y$ Hughes* ${ }^{1}{ }^{1} \mathrm{C}$ Chung, ${ }^{1,2,3}$ Zablotska-Manos, ${ }^{1,2,3} \mathrm{D}$ Lewis. ${ }^{1}$ Western Sydney Sexual Health Centre, Parramatta, Australia; ${ }^{2}$ Westmead Clinical School, Westmead, Australia; ${ }^{3}$ Marie Bashir Institute for Infectious Diseases and Biosecurity, Westmead, Australia

\subsection{6/sextrans-2021-sti.400}

Background Treponema pallidum subsp. pallidum infection (TP) may be diagnosed either directly by dark-field microscopy (DFM) or polymerase chain reaction (PCR) assay, or indirectly by serology. TP PCR and serology are currently the mainstay of laboratory-based diagnosis due to technical challenges associated with DFM. We describe the performance of TP PCR and serology in the diagnosis of primary syphilis at Western Sydney Sexual Health Centre (WSSHC).

Methods This is a retrospective study of primary syphilis cases diagnosed at WSSHC over a five-year period (2015-2019). Medical case records were reviewed to extract laboratory results. Patients without a prior history of syphilis were screened with either an enzyme-linked immunosorbent total antibody assay (EIA, WSSHC-associated laboratory) or a 